\title{
Diagnóstico y manejo de la hernia paraduodenal*
}

\author{
Drs. DAVID A. MATEO DE ACOSTA A. ${ }^{1}$, ABEL ENRIQUE BELLO ${ }^{2}$, LESTER DE LEÓN², \\ DIEGO GERARDO VÁZQUEZ S. ${ }^{3}$, JHONELLE ANDREA WAISSBLUTH G. ${ }^{4}$ \\ 1 Department of Surgery University of Illinois College of Medicine at Chicago Metropolitan Group Hospitals, \\ Chicago, Illinois, U.S.A. \\ 2 Departamento de Cirugía, North Eastern Ohio Colleges of Medicine, Northside Medical Center, \\ Forum Health - Western Care Reserve System, Youngstown, Ohio. U.S.A. \\ 3 Departamento de Cirugía de Colon y Recto, Universidad Nacional Autónoma de México, Hospital General \\ de México (SSA). Ciudad de México, México. \\ 4 Universidad Mayor, Escuela de Medicina Santiago, Chile.
}

\begin{abstract}
Diagnosis and management of paraduodenal hernia

Internal hernias cause $1 \%$ of intestinal obstructions with aproximately $50 \%$ of these hernias been paraduodenal hernias. Paraduodenal hernias are the product of a malrotation of the midgut during embryogenesis or a non-fusion of the peritoneal folds. These type of internal hernia are more common in males and on the left side of the abdomen. The usual presentation age is in the $4^{\text {th }}$ decade of life. The clinical presentation of these patients is non specific and varies, ranging from mild dyspepsia to intestinal perforation and septic shock. The radiographic studies of these patients show a clumping of the intestinal loops on the upper quadrant of the abdomen with an image of abdominal compartamentalization. The surgical management of these patients should follow three simple principles. The intestines should be reduced, their perfusion and viability must be verified and the hernia sack entrance should be repaired with interrupted non absorbable sutures.

Key words: Paraduodenal hernia, mesocolic hernia, internal hernia, intestinal malrotation, small bowel obstruction, laparoscopic repair.
\end{abstract}

\section{Resumen}

Las hernias internas causan el 1\% de las obstrucciones intestinales. De estas, aproximadamente la mitad son causadas por hernias paraduodenales. Las hernias paraduodenales son producto de una embriogénesis defectuosa, donde el intestino no rota o los pliegues mesentéricos no se fusionan adecuadamente. Estas hernias son más comunes en el género masculino, del lado izquierdo y por lo general se diagnostican en la cuarta década de la vida. La presentación clínica es inespecífica con manifestaciones que varían desde la dispepsia

\footnotetext{
* Recibido el 29 de Junio de 2010 y aceptado para publicación el 20 de Agosto de 2010

Este Trabajo no ha sido apoyado por ninguna institución o fuente económica tipo "Grant".

Este Trabajo no presenta conflicto de intereses.

Los autores no son parte de empresas farmacéuticas ni presentan conflicto de intereses con respecto al tema.

Correspondencia: Dr. David A. Mateo de Acosta

Advocate Illinois Massonic Medical Center. 836 West Wellington Avenue - Room 4807, Chicago, Illinois 60657 USA.

drdavidmateodea@yahoo.com.mx
} 
hasta la perforación intestinal y el choque séptico. Los estudios de imagen muestran el agrupamiento de las asas intestinales en el abdomen superior dando una imagen de compartamentalización de la cavidad abdominal. Para el manejo quirúrgico de estos pacientes se debe reducir el intestino herniado, verificar que este sea viable y posea una adecuada perfusión y reparar el orificio herniario con material no absorbible.

Palabras clave: Hernia paraduodenal, hernia mesocólica, hernia interna, malrotación intestinal, obstrucción intestinal, manejo laparoscópico.

\section{Introducción}

Las hernias internas causan el $0,9 \%$ de las obstrucciones intestinales y de estas el 53\% son hernias paraduodenales, con más de 500 casos reportados en la literatura mundial. La edad media de diagnóstico es a los 38,5 años de edad y son tres veces más comunes en el paciente masculino ${ }^{1}$. El $75 \%$ de las hernias paraduodenales son izquierdas. La presentación en el paciente adulto varía desde asintomático o leve dispepsia hasta la perforación intestinal y la sépsis abdominal ${ }^{1}$.

El riesgo de desarrollar una obstrucción y perforación intestinal durante la vida es del $50 \%$ en estos pacientes y acarrea una mortalidad del $20 \%$ al $50 \%$, dependiendo la serie consultada ${ }^{2}$.

\section{Reseña histórica}

Las primeras contribuciones a la descripción de las fosas paraduodenales datan de 1857, cuando Treitz describió la fosa "duodenoyeyunal"3. Posteriormente, Landzert describió una segunda fosa, que llamó la fosa paraduodenal, hoy conocida como fosa de Landzert. Broesike, Gruber, Waldeyer y Jonnesco, aportaron significativamente al conocimiento de la anatomía y el desarrollo de las fosas paraduodenales, describiendo un sin número de fosas, $\mathrm{y}$ recesos peritoneales que conllevaron a una era de confusión anatómica, durante la primera mitad del siglo pasado.

En 1917, Desjardins, describió la fosa paraduodenal superior como aquella delimitada por una banda delgada del peritoneo que recorre un trayecto en el plano posteroinferior al ligamento de Treitz, originándose a la izquierda del ángulo duodeno-yeyunal, con una longitud de $1 \mathrm{a} 2 \mathrm{~cm}$ y anclándose a la izquierda. El apex de esta fosa es el borde inferior del páncreas, su borde derecho es la cuarta porción del duodeno, y el izquierdo la fusión del peritoneo visceral y parietal a la altura de la vena mesentérica inferior. Desjardins, describió una segunda fosa inferior o de Treitz, como aquella definida en un plano inferior al orificio de entrada de la fosa paraduodenal superior y delimitada anteriormente por la reflexión peritoneal de la cuarta porción del duodeno, posteriormente por la hoja parietal del peritoneo de la cuarta porción del duodeno, en el lado derecho por el duodeno y en el izquierdo por la línea de fusión entre esta reflexión y el peritoneo parietal de la cuarta porción del duodeno con una profundidad de hasta $5 \mathrm{~cm}$.

Con el diagnóstico preoperatorio de la primera hernia paraduodenal en el año 1930 por Taylor ${ }^{5}$, la descripción detallada de la anatomía de la región por Desjardin y los estudios de Andrews ${ }^{6}$ sobre el mecanismo embriológico de esta patología, se inició una era donde el cirujano tendría a la hernia paraduodenal como un diagnóstico diferencial del dolor abdominal agudo y la obstrucción intestinal. En 1941, Cogswell describió la hernia paraduodenal derecha como una entidad aparte definiéndola como la incarceración de la mayor parte del intestino delgado posterior a los pliegues mesocólicos a través de un defecto en el área posterior al ángulo duodeno-yeyunal ${ }^{7}$, concordando con la definición de Moynihan de $1906^{8}$.

\section{Embriogénesis de la hernia paraduodenal}

La primera descripción de la rotación intestinal en el período embrionario fue realizada por Dott en 1923, estableciendo conceptos aceptados hasta la actualidad $^{9}$. Dott, definió al intestino medio como aquella porción del intestino irrigada por la arteria mesentérica superior (AMS), que incluye al duodeno distal, yeyuno, íleon, ciego, al colon ascendente y al transverso en sus $2 / 3$ partes proximales. La porción pre-arterial se desarrollará para formar el duodeno distal, el yeyuno y el íleon proximal y está localizada cefálica al conducto onfalo-mesentérico, mientras que la post arterial formará el íleon terminal, el ciego, el colon ascendente y el colon transverso en sus $2 / 3$ partes proximales y se localiza caudal al conducto onfalo-mesentérico.

El desarrollo del intestino medio involucra tres fases de rotación. En la quinta semana de gestación, el intestino medio crece hasta no poder ser contenido en la cavidad abdominal y sale al saco vitelino. Posteriormente, en la décima semana de gestación la cavidad abdominal es lo suficientemente amplia para albergar al intestino medio, y este regresa rotando $90^{\circ}$ en sentido contrario a las manecillas del reloj sobre su eje de suspensión, el mesenterio dorsal y la 
AMS. En esta fase el segmento pre-arterial ocupa el lado derecho de la cavidad abdominal del embrión y el post-arterial el izquierdo. Si la rotación del intestino se ve frenada en esta fase se da un fenómeno denominado "no rotación" lo cual conlleva a que la cuarta porción del duodeno, el yeyuno y el íleon se encuentran en el lado derecho del abdomen, posterior a la reflexión peritoneal, mientras que el ciego y el colon se encuentran en el lado izquierdo de la cavidad abdominal (Figura 1). Este fenómeno de "no rotación" es el causante de la hernia paraduodenal derecha.

La segunda fase de la rotación del intestino medio se da entre la décima y undécima semanas de gestación, e involucra la rotación del segmento pre-arterial en sentido contrario de las manecillas del reloj en $180^{\circ}$ adicionales para un total de $270^{\circ}$ de rotación sobre su eje. Inicialmente esta rotación es hacia lo posterior y hacia la izquierda de la AMS con el segmento pre-arterial en el lado izquierdo de la cavidad abdominal y el segmento post- arterial, que también rota $180^{\circ}$ adicionales, en el cuadrante superior derecho de la cavidad abdominal.

Cuando el segmento pre-arterial termina su rotación, se encuentra en el cuadrante inferior izquierdo

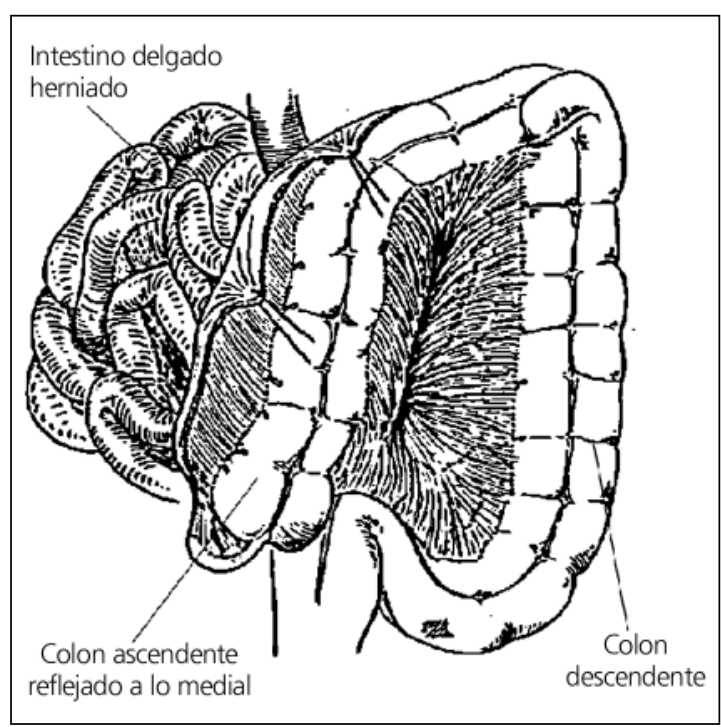

Figura 1. Fenómeno de No Rotación-Hernia Paraduodenal Derecha. En esta figura se observa el hallazgo transoperatorio del fenómeno de no rotación o arresto en la primera fase de la rotación intestinal normal. La fascia de Told derecha (ascendente) ha sido seccionada y el colon ascendente se ha rechazado sobre el eje de la línea media para exponer el intestino delgado. Este ya no se encuentra herniado. Al reparar este defecto se debe reparar el orificio de la hernia en la raíz del mesenterio para evitar que se repita este fenómeno. del abdomen cubierto por la raíz del mesenterio y la reflexión peritoneal. En este momento el segmento pre-arterial se invagina en un área débil del mesocolon descendente definida por el arco vascular que describen la rama ascendente de la arteria mesentérica inferior (AMI) y la vena mesentérica inferior (VMI) en su trayecto ascendente para desembocar en la vena esplénica en el triángulo venoso de Treitz. (Figuras 2a y 2b).

En la tercera fase de la rotación intestinal, se fusionan y adosan las capas del peritoneo posterior y la pared abdominal con la raíz del mesenterio. Si durante esta fase los segmentos del intestino medio no han culminado su migración y rotación, y los espacios peritoneales laxos que han permitido la migración intestinal, no se obliteran, se crea un orificio herniario y un saco que se cubre con peritoneo.

\section{Presentación clínica}

La presentación clínica típica de la hernia paraduodenal no es clara. Los pacientes se comportan de manera individual y errática. Aquellos pacientes con hernias pequeñas donde el asa intestinal involucrada se reduce espontáneamente presentan manifestaciones poco significativas como la dispepsia. Sin embargo, aquellos pacientes con grandes sacos herniarios que contienen más del $40 \%$ del intestino delgado y donde el asa intestinal involucrada se estrangula y perfora, presentan datos de abdomen agudo, obstrucción intestinal y choque séptico.

En el $50 \%$ de los pacientes, el cuadro es el de una obstrucción intestinal crónica e intermitente, con síntomas que duran de 6 meses a 30 años, con un promedio de 5 años. Estos, presentan episodios recurrentes de náuseas, vómitos y dolor abdominal difuso generalizado. En el transoperatorio, estos pacientes muestran múltiples adherencias y compartamentalización de la cavidad abdominal que ocasiona retraso del vaciamiento gástrico y aumenta la presión de las asas que se agrupan alrededor del orificio herniario por el cual deben transitar. Al ser evaluados en períodos libres de sintomatología, estos pacientes no muestran hallazgos clínicos, de laboratorio ni de imagen significativos por lo cual un gran porcentaje de ellos son manejados como trastornos funcionales o psicosomáticos. Es importante subrayar la necesidad del cirujano y del gastroenterólogo, de mantener en mente a la hernia paraduodenal como un diagnóstico diferencial, en aquellos pacientes que reportan dolor abdominal post prandial, exacerbado por esfuerzo o por la posición erecta y que a su vez es aliviado al ingerir porciones más pequeñas de alimentos y con un menor contenido de fibra ${ }^{1}$. 

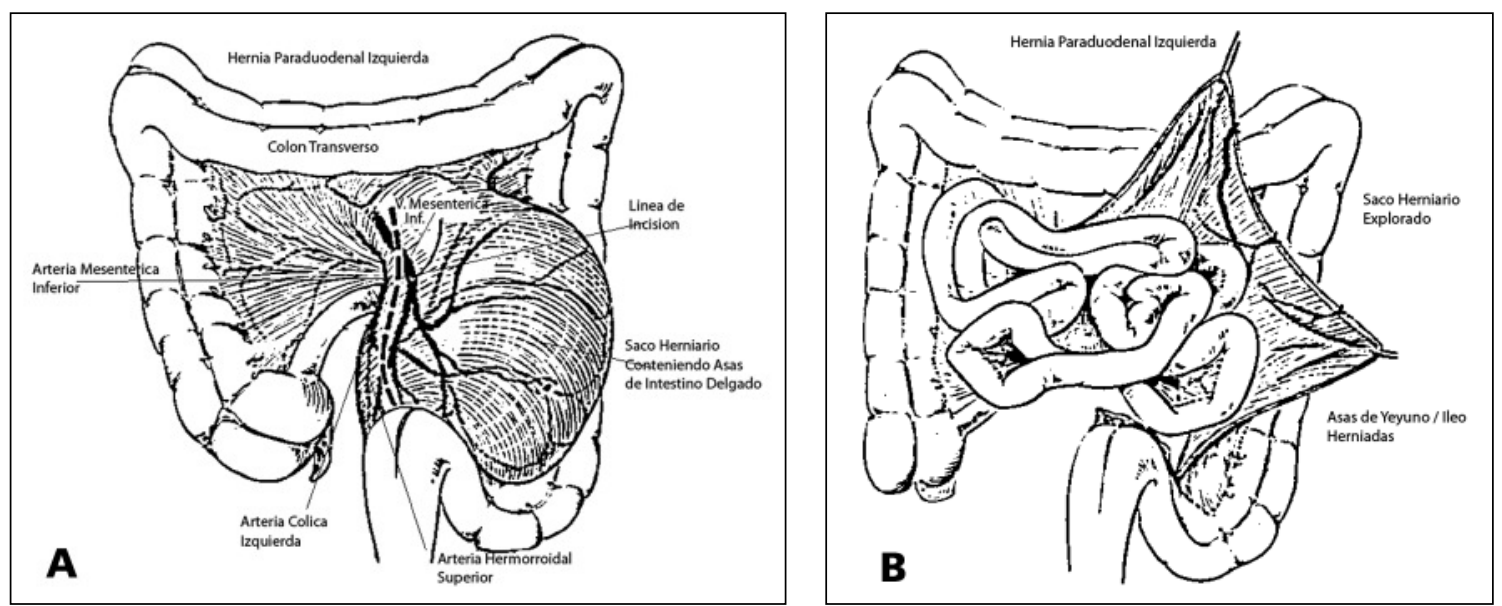

Figura 2. Hallazgo Intraoperatorio de la Hernia Paraduodenal Izquierda. A: En esta figura se observa el segmento prearterial del intestino medio contenido en un saco herniario al haberse invaginado por el mesocolon descendente debajo del arco definido por la rama descendente de la arteria mesentérica inferior y la vena mesentérica inferior en su curso cefálico. Se observa el íleon terminal como pasa por un orificio del peritoneo que se fusionó en la tercera etapa del desarrollo sobre este describiendo el arco vascular previamente mencionado. B: En esta figura se observa el contenido del saco herniario, el segmento pre arterial del intestino medio, al ser expuesto por una incisión paralela a la arcada vascular descrita en la Figura 2a.

El $50 \%$ restante de los pacientes con hernias paraduodenales, se manifiestan de manera aguda. Estos requieren descompresión quirúrgica inmediata ya que presentan obstrucciones intestinales de asa cerrada que no remiten al manejo médico y corren riesgo de perforación intestinal y de desarrollar choque séptico ${ }^{10,11}$. La exploración física de hasta el $18 \%$ de los pacientes con hernia paraduodenal izquierda, muestra una masa en el cuadrante inferior izquierdo, dolorosa y presenta datos de irritación peritoneal, durante los períodos de sintomatología ${ }^{12,13}$.

\section{Hallazgos radiográficos específicos}

\section{Estudios simples y contrastados}

Los estudios de imagen son parte esencial del diagnóstico de los pacientes en los que se sospecha la presencia de una hernia paraduodenal. El estudio radiográfico simple del abdomen muestra signos inespecíficos comunes a todos los cuadros de obstrucción intestinal mecánica, como la presencia de niveles hidro-aéreos y de asas intestinales con poca movilidad. Los signos que orientan al diagnóstico de hernia paraduodenal son la ausencia de contenido intestinal en la pelvis y la acumulación de las asas intestinales en un área circunscrita del abdomen limitadas al hemiabdomen superior. Esta conglomeración no es móvil y no cambia de posición con la manipulación abdominal ni los cambios de posición del paciente ${ }^{14}$.
El diagnóstico de la hernia paraduodenal puede ser confuso y elusivo por lo que se recomienda que, si la condición del paciente lo permite, se realice un tránsito intestinal completo contrastado. Este muestra una imagen de encapsulación de las asas de intestino delgado en el hemiabdomen superior en el cuadrante en el que se encuentra la hernia y la ausencia de las interdigitaciones intestinales, dando una imagen radiográfica de una masa de bordes regulares y lisos. Este borde se denomina borde de Donelly y sirve para distinguir las asas atrapadas en el saco herniario de aquellas que se encuentran libres ${ }^{15}$.

El estudio contrastado del paciente con una hernia paraduodenal izquierda muestra aglomeración de asas de yeyuno en el cuadrante superior izquierdo laterales a la cuarta porción del duodeno, aunque se han reportado la presencia asas de yeyuno en el cuadrante superior derecho y la presencia de un asa de yeyuno pasando posterior y hacia la izquierda de la vena mesentérica inferior (VMI) y la arteria cólica izquierda (ACI). El intestino delgado ejerce un efecto de masa rechazando al colon transverso en su porción distal y la pared posterior del estómago.

La fosa de Waldeyer es un defecto en el mesenterio de la primera parte del yeyuno, posterior a la AMS e interior a la tercera porción del duodeno ${ }^{16}$. Cuando el intestino delgado se hernia a través de esta fosa, el estudio contrastado muestra que la tercera porción del duodeno nunca atraviesa la línea media y las asas intestinales se aglomeran lateral e 
inferior a la segunda porción del duodeno dentro de la mitad derecha del mesocolon transverso o posterior al mesocolon ascendente ${ }^{17}$.

\section{Papel de la tomografía computarizada}

La tomografía computarizada (TC) aporta datos conclusivos que ayudan al diagnóstico preoperatorio del paciente con hernia paraduodenal. Cuando la hernia es izquierda, la TC muestra un grupo de asas intestinales encapsuladas en una cavidad delimitada por el cuerpo y la cola del páncreas, y el estómago lateral a la porción ascendente del duodeno, hacia la izquierda del ligamento de Treitz ${ }^{18}$. El orificio de entrada a este saco herniario se localiza posterior o posteromedial al duodeno, y las asas intestinales herniadas producen un efecto de masa sobre el colon transverso y los vasos mesentéricos inferiores, los cuales son rechazados hacia la izquierda. Ocasionalmente se logran observar la AMI y la rama ascendente de la ACI en el borde antero-medial del saco herniario ${ }^{19}$.

Cuando la hernia es derecha, la TC muestra las asas intestinales herniadas hacia el retroperitoneo en el espacio retrogástrico, localizándose en un plano inferior y lateral a la porción descendente del duodeno. En estos pacientes es frecuente observar una asa aferente de yeyuno y una eferente de yeyuno o íleon, las cuales entran y salen respectivamente a través del orificio de entrada de la fosa mesentericoparietal de Waldeyer ${ }^{20}$.

En el año 2008, el Departamento de Radiología del Hospital Universitario de la Universidad Médica
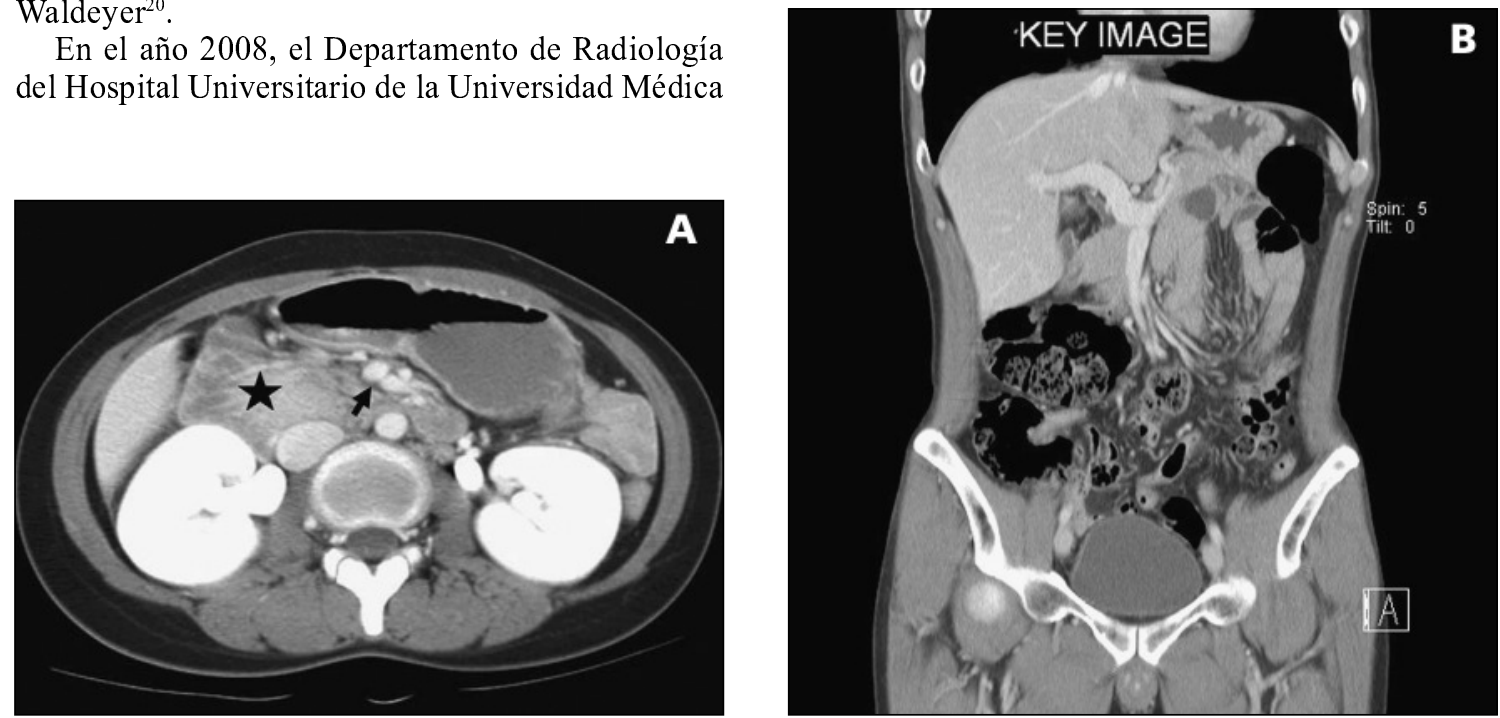

Figura 3. Hallazgo en Tomografía Computarizada de la hernia paraduodenal. A: Hallazgo en Tomografía Computarizada de la hernia paraduodenal derecha. En esta tomografía computarizada en corte axial se observa una conglomeración de asas de intestino delgado $(*)$ localizada en cuadrante superior derecho del abdomen posterior a la arteria mesentérica superior (flecha). B: Hallazgo en Tomografía Computarizada de la hernia paraduodenal derecha. En reconstrucción coronal de la tomografía se observan las asas intestinales en el cuadrante superior izquierdo del abdomen posterior e inferior al estómago y los vasos mesentéricos se observan ingurgitados y con aumento de su longitud debido al efecto de masa del intestino herniado. Cortesía de Guy-Seok Cho MD, PhD, Departamento de Cirugía Hospital Universitario de Soonchunhyang, República de Korea. 
a la cavidad abdominal se inspecciona y verifica la localización de la hernia paraduodenal y su relación con los vasos mesentéricos. Al realizar el abordaje de una hernia paraduodenal derecha, las asas intestinales se encuentran herniadas a través de la fosa de Waldeyer posterior a los vasos mesentéricos superiores. Se procede a la reducción digital del con-

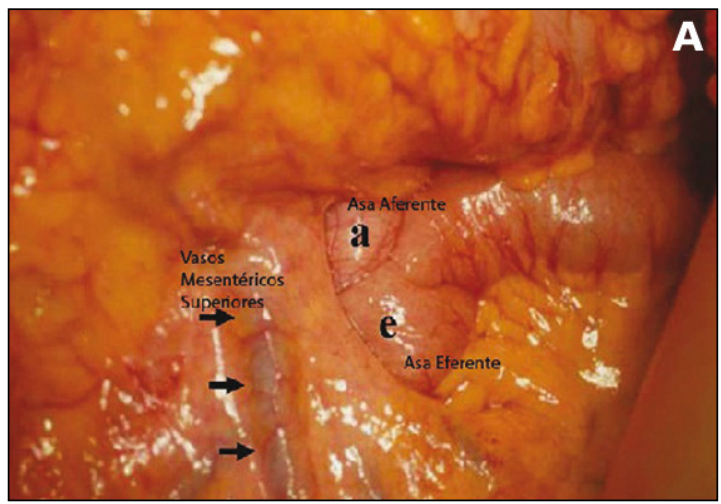

Figura 4. Hallazgos Transoperatorios de un paciente con hernia paraduodenal derecha. A: En esta fotografía laparoscópica se observa el asa aferente (a) y el asa eferente (e) del fragmento de intestino herniado a través de la Fosa de Waldeyer posterior a los vasos mesentéricos superiores (flechas). B: En esta fotografía tomada en una reparación abierta de una hernia paraduodenal derecha se observa una vista lateral del orificio de entrada dela fosa de Waldeyer. La pinza de disección rechaza hacia lo superior a los vasos mesentéricos superiores y se observa el asa aferente del intestino delgado herniado a través del orificio de entrada. Cortesía de Guy-Seok Cho MD, $\mathrm{PhD}$, Departamento de Cirugía Hospital Universitario de Soonchunhyang, República de Korea.

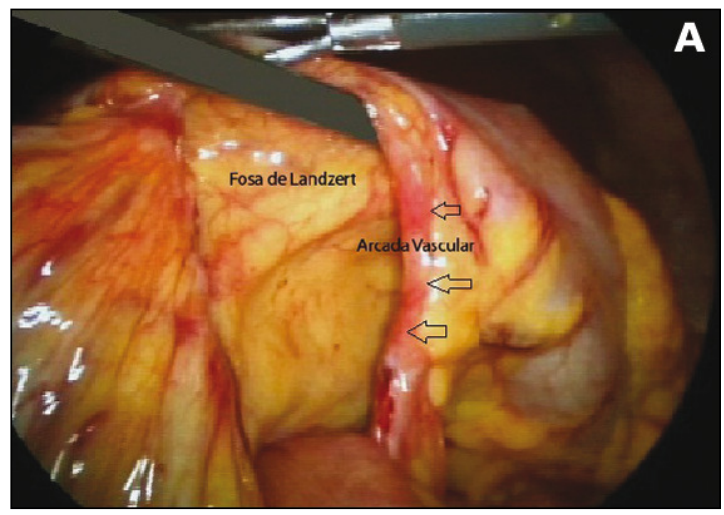

tenido intestinal herniado. Si es necesario se puede ampliar el orificio de entrada de la fosa de Waldeyer con cuidado de no lesionar los vasos mesentéricos superiores. Una vez reducido el contenido del saco herniario se repara el orificio de entrada a la fosa de Waldeyer con puntos simples de un material no absorbible como la seda ${ }^{22}$.

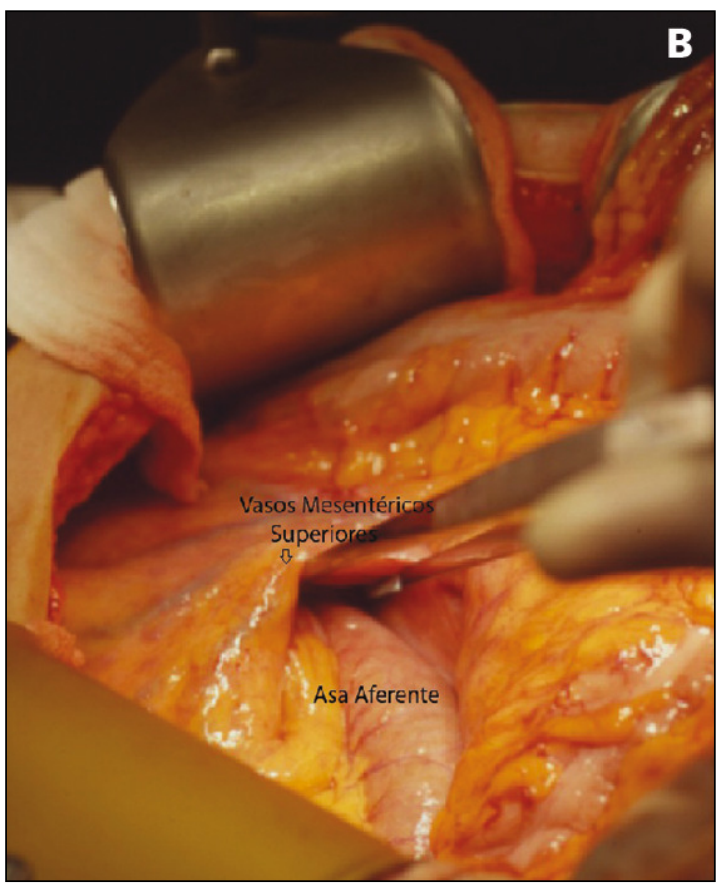

Figura 5. Fotografía laparoscópica de la reparación de una hernia paraduodenal izquierda. A: Se observa la vista laparoscópica del hemi-abdomen superior, el orificio herniario se observa a la izquierda del ligamento de Treitz y medial a la vena mesentérica inferior y la arteria cólica izquierda (flechas). B: El orificio herniario se ha reparado totalmente con nudos intracorpóreos (*). Cortesía de Guy-Seok Cho MD, PhD, Departamento de Cirugía Hospital Universitario de Soonchunhyang, República de Korea. 


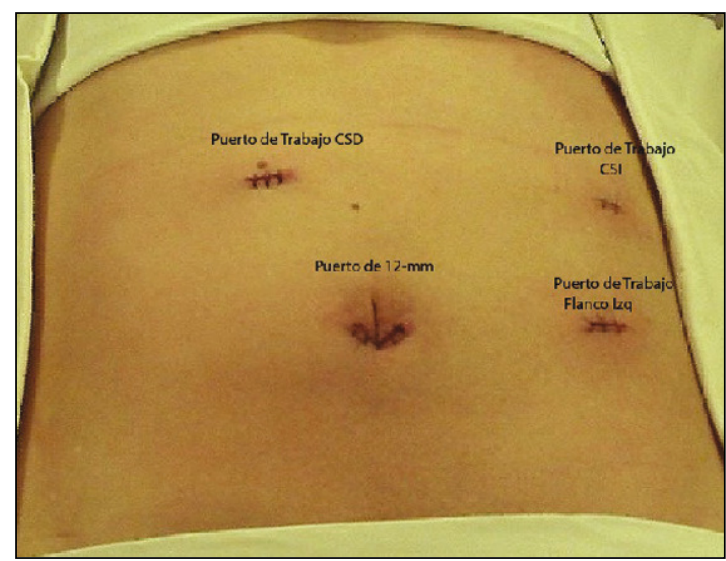

Figura 6. Vista Postoperatoria de la colocación de los puertos laparoscópicos en un paciente para la reparación de una hernia duodenal derecha. Observamos un puerto de $12 \mathrm{~mm}$ en el sitio de infraumbilical correspondiente al orificio de entrada de la cámara laparoscópica asi como los tres puertos de trabajo de $5 \mathrm{~mm}$. Cortesía de Guy-Seok Cho MD, PhD, Departamento de Cirugía hospital Universitario de Soonchunhyang, República de Korea.

Cuando el hallazgo quirúrgico es el de una hernia paraduodenal izquierda, el intestino herniado se debe disecar del orificio de entrada de la fosa de Landzert, inferior a la arcada vascular descrita por la VMI en su trayecto ascendente y la ACI. Si es necesario se puede ampliar el orificio de entrada dividiendo la VMI. Posterior a la reducción del intestino, se repara el defecto de entrada con puntos simples de material no absorbible como la seda ${ }^{23}$.

Actualmente, el manejo laparoscópico electivo de la hernia paraduodenal ha sido propuesto por varios grupos, quienes han reportado sus resultados. Este sigue principios similares al manejo tradicional. El procedimiento se realiza con el paciente en posición de Trendelemburg invertida. El pneumoperitoneo se induce, con la introducción de un trócar de 12 $\mathrm{mm}$ en la posición infraumbilical, hasta llegar a una presión de $12 \mathrm{mmHg}$. A través de este puerto se introduce el laparoscopio y se examina la cavidad abdominal. Si la hernia es izquierda se observará el orificio de entrada a la fosa de Landzert inferior y a la izquierda del ligamento de Treitz y el yeyuno proximal herniado a través de este. Si la hernia es derecha se observará el yeyuno herniándose a través de orificio de entrada de la fosa de Waldeyer. De ser seguro el procedimiento laparoscópico, es decir, si se ha comprobado la viabilidad del intestino y el adecuado flujo vascular de este, se procede a colocar 3 puertos de trabajo de $5 \mathrm{~mm}$ en el cuadrante superior derecho, superior izquierdo y en el inferior izquierdo respectivamente. En la hernia izquierda se observará el saco localizado posterior al estómago en el retroperitoneo, mientras que si es derecha, se observará las asas de intestino herniadas en el cuadrante superior derecho rechazando al colon transverso y los vasos mesentéricos superiores se encontrarán en el borde anterior del límite de este saco. Se procede a la reducción del intestino herniado con graspers intestinales no traumáticos sin incidir el orificio de entrada de la fosa. Posterior a la reducción, se colocan puntos simples intracorpóreos de material no absorbible cerrando el orifico de entrada a la fosa y se cierran las incisiones de los puertos de trabajo y del laparoscopio respectivamente.

El manejo laparoscópico de estas patologías es cómodo y seguro. En el paciente con una hernia paraduodenal el intestino se encuentra herniado, usualmente antes de los primeros $25 \mathrm{~cm}$ del ángulo duodeno yeyunal ${ }^{14}$. Esto reduce el contenido intrabodominal de asas intestinales distendidas facilitando la visibilidad y el abordaje laparoscópico. La distensión abdominal y la presencia de un gran contenido intestinal son factores que llevan a la conversión del procedimiento laproscópico a uno abierto ${ }^{22-24}$. Suter $^{22}$, reporta que un diámetro intestinal mayor a $4 \mathrm{~cm}$ es un factor significativo para la conversión del procedimiento laparoscópico a uno abierto. Así mismo el grupo de Fukunaga ${ }^{25}$, concluye que la ausencia de dilatación y gangrena en las asas intestinales, es un factor primordial para el abordaje laparoscópico de la hernia paraduodenal.

\section{Referencias}

1. Bernardi RS. Paraduodenal hernias [Review]. Surg Gynecol Obstet. 1981;152:99-100.

2. Tong RS, Shomik S, Tjandra J. Left Paraduodenal hernia. Anz J Surg. 2002;72:69-71.

3. Treitz, W. Hernia retroperitonealis. Ein Beitrag zur Geschichte inneres Hernien. Prag, Credner, 1857, 150 pp.

4. Desjardins AU. Left Paraduodenal Hernia. Rochester, Minnesota. 1918, 195-201 pp.

5. Taylor J. The x-ray Diagnosis of Right Paraduodenal Hernia. Brit J Surg. 1930;17:630.

6. Andrews E. Duodenal Hernia, a Misnomer. Surg Gynec Obstet. 1923;37:740.

7. Cogswell HD, Thomas CA. Right Paraduodenal Hernia. Ann Surg. 1941:114:1035-41.

8. Moynihan BGA. On Retroperitoneal Hernia. New York, William Wood and Co., 1906.

9. Dott NM. Anomalies of intestinal rotation: their embryology and surgical aspects with report of 5 cases. Br J Surg. 1923;11:251-86.

10. Freud H, Perlaztky Y. Small paraduodenal hernias. Arch Surg. 1977;112:1180-3.

11. Bartlet MK, Wang C, Williams WH. The Surgi- 
DIAGNÓSTICO Y MANEJO DE LA HERNIA PARADUODENAL

cal Treatment of Paraduodenal hernia. Ann Surg. 1968:168:249-54.

12. Hirasaki S, Koide N, Shima Y. Unusual variant of left paraduodenal hernia herniated into the mesocolic fossa leading to jejunal strangulation. J Gastroenterol. 1998;33:734-8.

13. Uemastu T, Kitamura H, Iwase M. Laparoscopic repair of a paraduodenal hernia. Surg Endosc. 1998;12:50-2.

14. Myers MA. Paraduodenal hernia, Radiologic and arteriographic diagnosis. Radiology 1970;95:29-37.

15. Donelly LF, Rencken IO, deLorimier AA, Gooding CA. Left paraduodenal hernia leading to ileal obstruction. Pediatr Radiol. 1996:26:534-6.

16. Blachar A, Federle MP. Internal hernia: an increasingly common cause of small bowel obstruction. Semin Ultrasound CT MR. 2002;23:174-183.

17. Martin LC, Merkle EM, Thompson WM. Abdominal Internal Hernias: Radiographic Findings. Contempor Diagnost Radiol. 2005;28:1-6.

18. Millar PA, Mezwa DG, Feczko PJ. Imaging of abdominal hernias. Radiographics 1995;15:333-47.

19. Okino Y, Kiyosue H, Mori H. Root of the small bowel mesentery: correlative anatomy and CT features of pathologic conditions. Radiographics 2001;21:1475-90.

20. Yeoman LJ, Patel AG, Michell MJ. Case report: computed tomography appearances in a right paraduodenal hernia. Clin Radiol. 1994;49:898-900.

21. Chien-Heng L, Yung-jen H, Wei-Ching L. Preoperative Diagnosis of Right Paraduodenal Hernia by Multidetector Computed Tomography. J Formos Med Asoc. 2008;107:500-4.

22. Suter M, Zermatten P, Halkic N. Laparoscopic management of mechanical small bowel obstruction: are there predictors of success or failure? Surg Endos. 2000;14:478-83.

23. Kirshtein B, Roy-Shapira A, Lantsberg L. Laparoscopic management of acute small bowel obstruction. Surg Endosc. 2005;19:464-7.

24. Khalaileh A, Schlager A, Bala M, Abugazala S, Elazary R, Rivkind AL, et al. Left Laparoscopic paraduodenal hernia repair. Surg Endosc. 2010;24:1486-9.

25. Fukunaga M, Jidokoro A, Iba T. Laparoscopic Surgery for left paraduodenal hernia. J Laparoendosc Adv Surg Tech A. 2004;14:111-5. 